\title{
New Unconditionally Stable Algorithms to Solve the Time-Dependent Maxwell Equations
}

\author{
J.S. Kole, M.T. Figge, and H. De Raedt \\ Centre for Theoretical Physics and Materials Science Centre \\ University of Groningen, Nijenborgh 4, \\ NL-9747 AG Groningen, The Netherlands \\ E-mail: j.s.kole@phys.rug.nl, m.t.figge@phys.rug.nl, deraedt@phys.rug.nl \\ http://rugth30.phys.rug.nl/compphys
}

\begin{abstract}
We present a family of unconditionally stable algorithms, based on the Suzuki product-formula approach, that solve the timedependent Maxwell equations in systems with spatially varying permittivity and permeability. Salient features of these algorithms are illustrated by computing the density of states and by simulating the propagation of light in a two-dimensional photonic material.
\end{abstract}

\section{Introduction}

The Maxwell equations describe the evolution of electromagnetic (EM) fields in space and time [1]. They apply to a wide range of different physical situations and play an important role in a large number of engineering applications. In many cases, numerical methods are required to solve Maxwell's equations, either in the frequency or time domain. For the time domain, a well-known class of algorithms is based on a method proposed by Yee [2] and is called the finite-difference timedomain (FDTD) method. The FDTD method has matured during past years and various algorithms implemented for specific purposes are available by now $[3,4]$. These algorithms owe their popularity mainly due to their flexibility and speed while at the same time they are easy to implement. A limitation of Yee-based FDTD techniques is that their stability is conditional, depending on the mesh size of the spatial discretization and the time step of the time integration [3].

In this paper we describe the recently introduced family of unconditionally stable algorithms that solve the time-dependent Maxwell equations (TDME) [5]. From the representation of the TDME in matrix form, it follows that the timeevolution operator of the EM fields is the exponential of a skew-symmetric matrix. This time-evolution operator is orthogonal. The key to the construction of rigorously provable unconditionally stable algorithms is the observation, that orthogonal approximations to this operator automatically yield unconditionally stable algorithms. The Lie-Trotter-Suzuki product formulae [6] provide the mathematical framework to construct orthogonal approximations to the timeevolution operator of the Maxwell equations. 


\section{Theory}

The model system we consider describes EM fields in a $d$-dimensional $(d=1,2,3)$ medium with spatially varying permittivity and/or permeability, surrounded by a perfectly conducting box. In the absence of free charges and currents, the EM fields in such a system satisfy Maxwell's equations [1]

$$
\begin{aligned}
& \frac{\partial}{\partial t} \mathbf{H}=-\frac{1}{\mu} \nabla \times \mathbf{E} \quad \text { and } \quad \frac{\partial}{\partial t} \mathbf{E}=\frac{1}{\varepsilon} \nabla \times \mathbf{H}, \\
& \operatorname{div} \varepsilon \mathbf{E}=0 \quad \text { and } \quad \operatorname{div} \mathbf{H}=0,
\end{aligned}
$$

where $\mathbf{H}=\left(H_{x}(\mathbf{r}, t), H_{y}(\mathbf{r}, t), H_{z}(\mathbf{r}, t)\right)^{T}$ and $\mathbf{E}=\left(E_{x}(\mathbf{r}, t), E_{y}(\mathbf{r}, t), E_{z}(\mathbf{r}, t)\right)^{T}$ denote the magnetic and electric field vector respectively. The permeability and the permittivity are given by $\mu=\mu(\mathbf{r})$ and $\varepsilon=\varepsilon(\mathbf{r})$. For simplicity of notation, we will omit the spatial dependence on $\mathbf{r}=(x, y, z)^{T}$ unless this leads to ambiguities. On the surface of the perfectly conducting box the EM fields satisfy the boundary conditions [1]

$$
\mathbf{n} \times \mathbf{E}=0 \quad \text { and } \quad \mathbf{n} \cdot \mathbf{H}=0,
$$

with $\mathbf{n}$ denoting the vector normal to a boundary of the surface. The conditions Eqs. (3) assure that the normal component of the magnetic field and the tangential components of the electric field vanish at the boundary [1]. Some important symmetries of the Maxwell equations (1)-(2) can be made explicit by introducing the fields

$$
\mathbf{X}(t)=\sqrt{\mu} \mathbf{H}(t) \quad \text { and } \quad \mathbf{Y}(t)=\sqrt{\varepsilon} \mathbf{E}(t) .
$$

In terms of the fields $\mathbf{X}(t)$ and $\mathbf{Y}(t)$, the TDME (1) read

$$
\frac{\partial}{\partial t}\left(\begin{array}{l}
\mathbf{X}(t) \\
\mathbf{Y}(t)
\end{array}\right)=\left(\begin{array}{cc}
0 & -\frac{1}{\sqrt{\mu}} \nabla \times \frac{1}{\sqrt{\varepsilon}} \\
\frac{1}{\sqrt{\varepsilon}} \nabla \times \frac{1}{\sqrt{\mu}} & 0
\end{array}\right)\left(\begin{array}{c}
\mathbf{X}(t) \\
\mathbf{Y}(t)
\end{array}\right) \equiv \mathcal{H}\left(\begin{array}{c}
\mathbf{X}(t) \\
\mathbf{Y}(t)
\end{array}\right)
$$

Writing $\Psi(t)=(\mathbf{X}(t), \mathbf{Y}(t))^{T}$, Eq. (5) becomes

$$
\frac{\partial}{\partial t} \Psi(t)=\mathcal{H} \Psi(t)
$$

It is easy to show that $\mathcal{H}$ is skew-symmetric, i.e. $\mathcal{H}^{T}=-\mathcal{H}$, with respect to the inner product $\left\langle\Psi \mid \Psi^{\prime}\right\rangle \equiv \int_{V} \Psi^{T} \cdot \Psi^{\prime} d \mathbf{r}$, where $V$ denotes the volume of the enclosing box. The formal solution of Eq. (6) is given by

$$
\Psi(t)=U(t) \Psi(0)=e^{t \mathcal{H}} \Psi(0),
$$

where $\Psi(0)$ represents the initial state of the EM fields. The operator $U(t)=$ $e^{t \mathcal{H}}$ determines the time evolution. By construction $\|\Psi(t)\|^{2}=\langle\Psi(t) \mid \Psi(t)\rangle=$ $\int_{V}\left[\varepsilon \mathbf{E}^{2}(t)+\mu \mathbf{H}^{2}(t)\right] d \mathbf{r}$, relating the length of $\Psi(t)$ to the energy density $w(t) \equiv$ 
$\varepsilon \mathbf{E}^{2}(t)+\mu \mathbf{H}^{2}(t)$ of the EM fields [1]. As $U(t)^{T}=U(-t)=U^{-1}(t)=e^{-t \mathcal{H}}$ it follows that $\langle U(t) \Psi(0) \mid U(t) \Psi(0)\rangle=\langle\Psi(t) \mid \Psi(t)\rangle=\langle\Psi(0) \mid \Psi(0)\rangle$. Hence the timeevolution operator $U(t)$ is an orthogonal transformation, rotating the vector $\Psi(t)$ without changing its length $\|\Psi\|$. In physical terms this means that the energy density of the EM fields does not change with time, as expected on physical grounds [1].

The fact that $U(t)$ is an orthogonal transformation is essential for the development of an unconditionally stable algorithm to solve the Maxwell equations. In practice, a numerical procedure solves the TDME by making use of an approximation $\tilde{U}(t)$ to the true time evolution $U(t)$. A necessary and sufficient condition for an algorithm to be unconditionally stable is that $\|\tilde{U}(t) \Psi(0)\| \leq\|\Psi(0)\|$. In other words, the length of $\Psi(t)$ should be bounded, for arbitrary initial condition $\Psi(t=0)$ and for any time $t[7]$. By chosing for $\Psi(0)$ the eigenvector of $\tilde{U}(t)$ that corresponds to the largest eigenvalue of $\tilde{U}(t)$, it follows that the algorithm will be unconditionally stable by construction if and only if the largest eigenvalue of $\tilde{U}(t)$ (denoted by $\|\tilde{U}(t)\|$ ) is less or equal than one [7]. If the approximation $\tilde{U}(t)$ is itself an orthogonal transformation, then $\|\tilde{U}(t)\|=1$ and the numerical scheme will be unconditionally stable.

\section{Unconditionally Stable Algorithms}

A numerical procedure that solves the TDME necessarily starts by discretizing the spatial derivatives. This maps the continuum problem described by $\mathcal{H}$ onto a lattice problem defined by a matrix $H$. Ideally, this mapping should not change the basic symmetries of the original problem. The underlying symmetry of the TDME suggests to use matrices $H$ that are real and skew-symmetric. Since formally the time evolution of the EM fields on the lattice is given by $\Psi(t+\tau)=$ $U(\tau) \Psi(t)=e^{\tau H} \Psi(t)$, the second ingredient of the numerical procedure is to choose an approximation of the time-step operator $U(\tau)$. A systematic approach to construct orthogonal approximations to matrix exponentials is to make use of the Lie-Trotter-Suzuki formula $[8,9]$

$$
e^{t\left(H_{1}+\ldots+H_{p}\right)}=\lim _{m \rightarrow \infty}\left(\prod_{i=1}^{p} e^{t H_{i} / m}\right)^{m},
$$

and generalizations thereof $[6,10]$. Applied to the case of interest here, the success of this approach relies on the basic but rather trivial premise that the matrix $H$ can be written as $H=\sum_{i=1}^{p} H_{i}$, where each of the matrices $H_{i}$ is real and skew-symmetric. The expression Eq. (8) suggests that

$$
U_{1}(\tau)=e^{\tau H_{1}} \ldots e^{\tau H_{p}},
$$

might be a good approximation to $U(\tau)$ if $\tau$ is sufficiently small. Most importantly, if all the $H_{i}$ are real and skew-symmetric, $U_{1}(\tau)$ is orthogonal by construction. Therefore, by construction, a numerical scheme based on Eq. (9) will be 
unconditionally stable. Using the fact that both $U(\tau)$ and $U_{1}(\tau)$ are orthogonal matrices, it can be shown that the Taylor series of $U(\tau)$ and $U_{1}(\tau)$ are identical up to first order in $\tau$ [11]. We will call $U_{1}(\tau)$ a first-order approximation to $U(\tau)$. The product-formula approach provides simple, systematic procedures to improve the accuracy of the approximation to $U(\tau)$ without changing its fundamental symmetries. For example the orthogonal matrix

$$
U_{2}(\tau)=U_{1}(-\tau / 2)^{T} U_{1}(\tau / 2)=e^{\tau H_{p} / 2} \ldots e^{\tau H_{1} / 2} e^{\tau H_{1} / 2} \ldots e^{\tau H_{p} / 2},
$$

is a second-order approximation to $U(\tau)[6,10]$. Suzuki's fractal decomposition approach [6] gives a general method to construct higher-order approximations based on $U_{1}(\tau)$ or $U_{2}(\tau)$. A particularly useful fourth-order approximation is given by $[6]$

$$
U_{4}(\tau)=U_{2}(a \tau) U_{2}(a \tau) U_{2}((1-4 a) \tau) U_{2}(a \tau) U_{2}(a \tau),
$$

where $a=1 /\left(4-4^{1 / 3}\right)$. The approximations Eqs. (9), (10), and (11) have proven to be very useful in many applications [9-19] and, as we show below, turn out to be equally useful for solving the TDME. From Eqs. (9)-(11) it follows that, in practice, an efficient implementation of the first-order scheme is all that is needed to construct the higher-order algorithms Eqs. (10) and (11).

\section{Implementation}

The basic ingredients of our approach will be illustrated for a one-dimensional (1D) system. A discussion of the two-dimensional (2D) and three-dimensional (3D) case are given in Ref. [5].

Maxwell's equations for a 1D system extending along the $x$-direction contain no partial derivatives with respect to $y$ or $z$ and also $\varepsilon$ and $\mu$ do not depend on $y$ or $z$. Under these conditions, the TDME reduce to two independent sets of first-order differential equations [1]. The solutions to these sets are known as the transverse electric (TE) mode and the transverse magnetic (TM) mode [1]. Restricting our considerations to the TM-mode, it follows from Eq. (5) that the magnetic field $H_{y}(x, t)=X_{y}(x, t) / \sqrt{\mu(x)}$ and the electric field $E_{z}(x, t)=$ $Y_{z}(x, t) / \sqrt{\varepsilon(x)}$ are solutions of

$$
\begin{aligned}
\frac{\partial}{\partial t} X_{y}(x, t) & =\frac{1}{\sqrt{\mu(x)}} \frac{\partial}{\partial x}\left(\frac{Y_{z}(x, t)}{\sqrt{\varepsilon(x)}}\right), \\
\frac{\partial}{\partial t} Y_{z}(x, t) & =\frac{1}{\sqrt{\varepsilon(x)}} \frac{\partial}{\partial x}\left(\frac{X_{y}(x, t)}{\sqrt{\mu(x)}}\right) .
\end{aligned}
$$

Note that in 1D the divergence of $H_{y}(x, t)$ and $E_{z}(x, t)$ is zero, hence Eqs. (2) are automatically satisfied. Using the second-order central-difference approximation to the first derivative with respect to $x$, we obtain

$$
\frac{\partial}{\partial t} X_{y}(i, t)=\frac{1}{\delta \sqrt{\mu_{i}}}\left(\frac{Y_{z}(i+1, t)}{\sqrt{\varepsilon_{i+1}}}-\frac{Y_{z}(i-1, t)}{\sqrt{\varepsilon_{i-1}}}\right),
$$




$$
\frac{\partial}{\partial t} Y_{z}(j, t)=\frac{1}{\delta \sqrt{\varepsilon_{j}}}\left(\frac{X_{y}(j+1, t)}{\sqrt{\mu_{j+1}}}-\frac{X_{y}(j-1, t)}{\sqrt{\mu_{j-1}}}\right),
$$

where the integer $i$ labels the grid points and $\delta$ denotes the distance between two next-nearest neighbor lattice points, as indicated in Fig. 1. For notational simplicity we will, from now on, specify the spatial coordinates through the lattice index $i$, e.g. $X_{y}(i, t)$ stands for $X_{y}(x=i \delta / 2, t)$. Following Yee [2] it is convenient to assign $X_{y}(i, t)$ and $Y_{z}(j, t)$ to the odd, respectively, even numbered lattice site, as shown in Fig. 1 for a grid of $n$ points. The equations (14) and (15) can now be combined into one equation of the form Eq. (6) by introducing the $n$-dimensional vector $\Psi(t)$ with elements

$$
\Psi(i, t)=\left\{\begin{array}{ll}
X_{y}(i, t)=\sqrt{\mu_{i}} H_{y}(i, t), & i \text { odd } \\
Y_{z}(i, t)=\sqrt{\varepsilon_{i}} E_{z}(i, t), & i \text { even }
\end{array} .\right.
$$

The vector $\Psi(t)$ describes both the magnetic and the electric field on the lattice points $i=1, \ldots, n$ and the $i$-th element of $\Psi(t)$ is given by the inner product $\Psi(i, t)=\mathbf{e}_{i}^{T} \cdot \Psi(t)$, where $\mathbf{e}_{i}$ denotes the $i$-th unit vector in the $n$-dimensional vector space. Using this notation, it is easy to show that $\Psi(t)=U(t) \Psi(0)$ with $U(t)=\exp (t H)$, where the matrix $H$ is represented by $H=H_{1}+H_{2}$ and

$$
\begin{aligned}
& H_{1}=\sum_{i=1}^{n-2} \beta_{i+1, i}\left(\mathbf{e}_{i} \mathbf{e}_{i+1}^{T}-\mathbf{e}_{i+1} \mathbf{e}_{i}^{T}\right), \\
& H_{2}=\sum_{i=1}^{n-2} \beta_{i+1, i+2}\left(\mathbf{e}_{i+1} \mathbf{e}_{i+2}^{T}-\mathbf{e}_{i+2} \mathbf{e}_{i+1}^{T}\right) .
\end{aligned}
$$

Here, $\beta_{i, j}=1 /\left(\delta \sqrt{\varepsilon_{i} \mu_{j}}\right)$ and the prime indicates that the sum is over odd integers only. For $n$ odd we have

$$
\frac{\partial}{\partial t} \Psi(1, t)=\beta_{2,1} \Psi(2, t) \quad \text { and } \quad \frac{\partial}{\partial t} \Psi(n, t)=-\beta_{n-1, n} \Psi(n-1, t),
$$

such that the electric field vanishes at the boundaries $\left(Y_{z}(0, t)=Y_{z}(n+1, t)=0\right.$, see also Fig. 1), as required by the boundary conditions Eqs. (3).

The representation of $H$ as the sum of $H_{1}$ and $H_{2}$ divides the lattice into odd and even numbered cells. Most important, however, both $H_{1}$ and $H_{2}$ are skewsymmetric block-diagonal matrices, containing one $1 \times 1$ matrix and $(n-1) / 2$ real, $2 \times 2$ skew-symmetric matrices. Therefore, according to the general theory

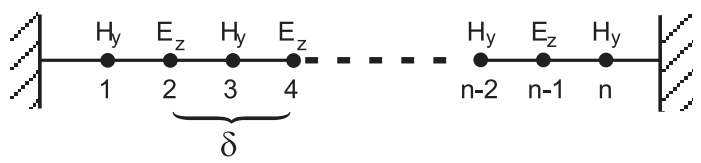

Fig. 1. Positions of the two TM-mode EM field components on the 1D grid. 


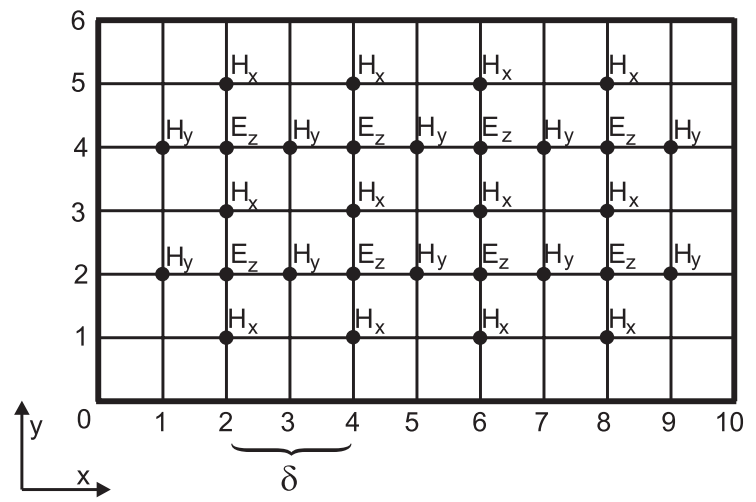

Fig. 2. Positions of the three TM-mode EM field components on the $2 \mathrm{D}$ grid for $n_{x}=9$ and $n_{y}=5$

given above, this decomposition of $H$ is suitable to construct an orthogonal first-order approximation

$$
U_{1}(\tau)=e^{\tau H_{1}} e^{\tau H_{2}}
$$

which is all that is needed to construct unconditionally stable second and higherorder algorithms. As the matrix exponential of a block-diagonal matrix is equal to the block-diagonal matrix of the matrix exponentials of the individual blocks, the numerical calculation of $e^{\tau H_{1}}$ (or $e^{\tau H_{2}}$ ) reduces to the calculation of $(n-1) / 2$ matrix exponentials of $2 \times 2$ matrices. The matrix exponential of a typical $2 \times 2$ matrix appearing in $e^{\tau H_{1}}$ or $e^{\tau H_{2}}$ is simply given by the rotation

$$
\exp \left[\alpha\left(\begin{array}{rr}
0 & 1 \\
-1 & 0
\end{array}\right)\right]\left(\begin{array}{l}
\Psi(i, t) \\
\Psi(j, t)
\end{array}\right)=\left(\begin{array}{rr}
\cos \alpha & \sin \alpha \\
-\sin \alpha & \cos \alpha
\end{array}\right)\left(\begin{array}{l}
\Psi(i, t) \\
\Psi(j, t)
\end{array}\right) .
$$

The implementation for $1 \mathrm{D}$ can be readily extended to $2 \mathrm{D}$ and $3 \mathrm{D}$ systems [5]. In 2D, the TDME (1) separate again into two independent sets of equations and the discretization of continuum space is done by simply re-using the 1D lattice introduced above. This is shown in Fig. 2 for the case of the 2D TM-modes. The construction automatically takes care of the boundary conditions if $n_{x}$ and $n_{y}$ are odd and yields a real skew-symmetric matrix $H$. Correspondingly, in 3D the spatial coordinates are discretized by adopting the standard Yee grid [2], which also automatically satisfies the boundary conditions Eqs. (3). A unit cell of the Yee grid is shown in Fig. 3.

We finally note, that in contrast to the $1 \mathrm{D}$ system the divergence of the EM fields is not conserved in $2 \mathrm{D}$ and 3D. Although the initial state (at $t=0)$ of the EM fields may satify Eqs. (2), time-integration of the TDME using $U_{k}(\tau)$ yields a solution that will not satisfy Eqs. (2). However, for an algorithm based on $U_{k}(\tau)$ the deviations from zero vanish as $\tau^{k}$, so that in practice these errors are under control and can be made sufficiently small. 


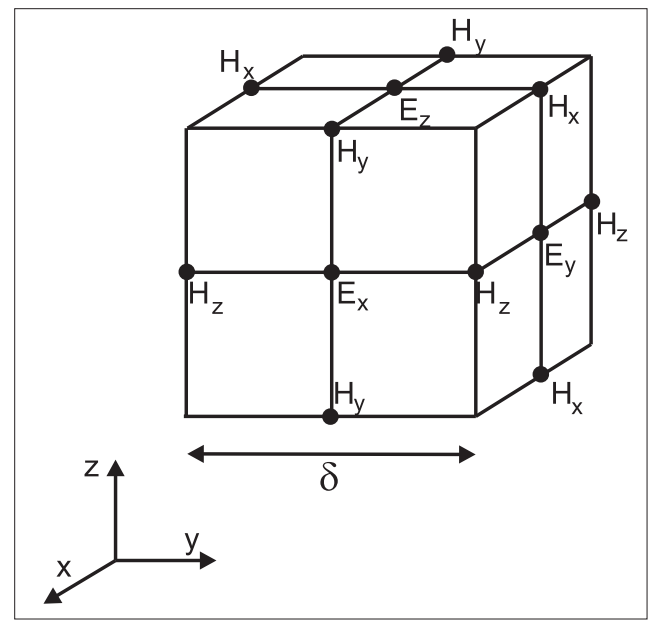

Fig. 3. Positions of the EM field components on the 3D Yee grid.

\section{Simulation Results}

We present simulation results for a 2D photonic bandgap (PBG) material. PBGs prohibit the propagation of EM fields in a range of frequencies that is characteristic for its structure [20]. A PBG is called absolute if it exists for any wave vector of the EM fields. The most common method used to compute a PBG employs a plane-wave expansion to solve the time-independent Maxwell equations (see e.g. [21]). This kind of calculation requires a Fourier transform of the unit cell of the dielectric structure which is for simplicity considered to be periodic. With our time-domain algorithm the existence of a PBG can be demonstrated with relative ease. It suffices to compute the spectrum of such a dielectric structure with a random initial state. If the spectrum is gapless there is no need to make additional runs. If there is a signature of a gap, it can be confirmed and refined by making more runs.

For numerical purposes we choose dimensionless quantities, where the unit of length is $\lambda$ and the velocity of light in vacuum, $c$, is taken as the unit of velocity. Then, time and frequency are measured in units of $\lambda / c$ and $c / \lambda$, respectively, while the permittivity $\varepsilon$ and permeability $\mu$ are measured in units of their corresponding values in vacuum. As an example we consider a system consisting of a dielectric material pierced by air-filled cylinders [22]. The geometry is taken to be a square parallelepiped of size $L=45.1$ that is infinitely extended in the $z$-direction and hence is effectively two-dimensional.

In Fig. 4 we present our results for the PBGs for both the transverse magnetic (TM) and transverse electric (TE) modes as a function of the filling fraction. The data have been generated by means of the algorithm $U_{4}(\tau)$ with a mesh size $\delta=0.1$ and a time step $\tau=0.1$. To compute the density of states (DOS) $\mathcal{D}(\omega)$ 


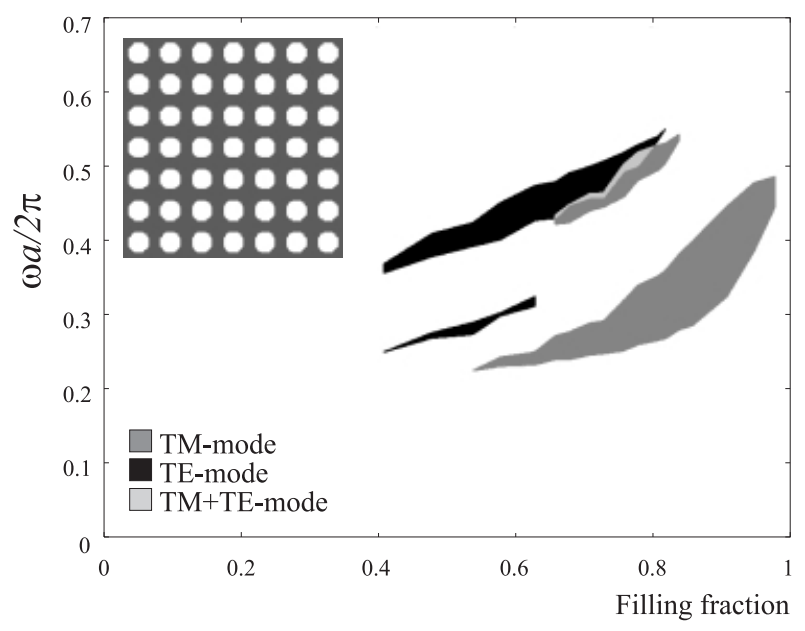

Fig. 4. Absolute photonic bandgaps of a dielectric material $(\varepsilon=11.4)$ pierced by airfilled cylinders. The largest overlap of the TM- and TE-mode gaps occurs at a filling fraction of approximately 0.77 .

we used only a single random initial state for the EM fields. The results shown in Fig. 4 are in good agreement with those presented in Ref. [22].

In Fig. 5 we study the propagation of time-dependent EM fields through the above described PBG material consisting of twelve unit cells. The PBG material is placed in a cavity which contains a point source that emits radiation with frequency $\omega$. The TDME were solved by the $U_{2}(\tau)$ algorithm with $\delta=0.1$ and $\tau=0.01$ in the presence of a current source. The snapshots show the absolute intensity $E_{z}^{2}$ of the TM-mode at $t=102.4$. The DOS of the PBG material is given in Fig. 6. We used the $U_{4}(\tau)$ algorithm with $\delta=0.1$ and $\tau=0.1$ in this computation and applied a time-domain algorithm to obtain the DOS [5,23].

The presence or absence of gaps in the DOS leads to qualitative changes in the transmitted (and reflected) intensities. Since a gap is present in the DOS at $\omega=1.89$, radiation with this frequency does not easily propagate through the (thin slice of) PBG material. On the other hand, the DOS has no gaps at $\omega=1.50$ and $\omega=2.50$, so that propagation of EM fields through the PBG material should be possible, as is indeed confirmed by Fig. 5 .

\section{Conclusion}

We have described a new family of algorithms to solve the time-dependent Maxwell equations. Salient features of these algorithms are:

- rigorously provable unconditional stability for 1D, 2D and 3D systems with spatially varying permittivity and permeability, 

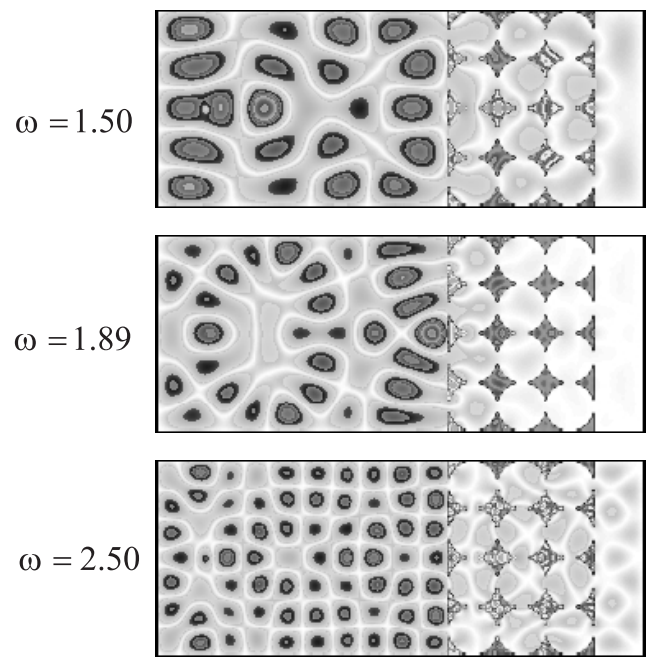

Fig. 5. Snapshot of the intensity $E_{z}^{2}$ at $t=102.4$. The size of the system is $30 \times 12.1$. A point source is located at $(6,6)$ (see Fig. 2), emitting radiation with frequency $\omega$.

- the use of a real-space (Yee-like) grid,

- the order of accuracy in the time step can be systematically increased without affecting the unconditional stability,

- the exact conservation of the energy density of the electromagnetic field,

- easy to implement in practice.

Although we believe there is little room to improve upon the time-integration scheme itself, for some applications it will be necessary to use a better spatial discretization than the most simple one employed in this paper. There is no fundamental problem to extend our approach in this direction and we will report on this issue and its impact on the numerical dispersion in a future publication.

We have presented numerical results for the density of states and the propagation of light in a two-dimensional photonic material. This illustrative example showed that our algorithms reproduce known results. The first of the above mentioned features opens up possibilities for applications to left-handed materials $[24,25]$ and we intend to report on this subject in the near future. In view of the generic character of the approach discussed in this paper, it can be used to construct unconditionally stable algorithms that solve the equations for e.g. sound, seismic and elastic waves as well.

\section{Acknowledgements}

This work is partially supported by the Dutch 'Stichting Nationale Computer Faciliteiten' (NCF). 


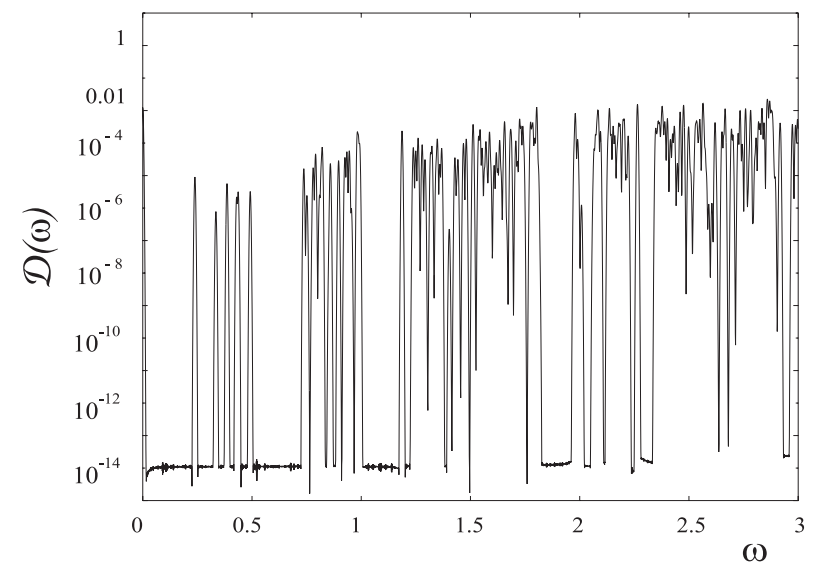

Fig. 6. Density of states $\mathcal{D}(\omega)$ of a sample of PBG material shown in Fig. 5. The size of the sample is $9.1 \times 12.1$ and the filling factor is 0.77 .

\section{References}

1. M. Born and E. Wolf, Principles of Optics, (Pergamon, Oxford, 1964).

2. K.S. Yee, IEEE Transactions on Antennas and Propagation 14, 302 (1966).

3. A. Taflove and S.C. Hagness, Computational Electrodynamics - The FiniteDifference Time-Domain Method, (Artech House, Boston, 2000).

4. See http://www.fdtd.org

5. J.S. Kole, M.T. Figge, and H. De Raedt, Phys. Rev. E 64, 066705 (2001).

6. M. Suzuki, J. Math. Phys. 26, 601 (1985); ibid 32400 (1991).

7. G.D. Smith, Numerical solution of partial differential equations, (Clarendon, Oxford, 1985).

8. H.F. Trotter, Proc. Am. Math. Soc. 10, 545 (1959).

9. M. Suzuki, S. Miyashita, and A. Kuroda, Prog. Theor. Phys. 58, 1377 (1977).

10. H. De Raedt and B. De Raedt, Phys. Rev. A 28, 3575 (1983).

11. H. De Raedt, Comp. Phys. Rep. 7, 1 (1987).

12. H. Kobayashi, N. Hatano, and M. Suzuki, Physica A 211, 234 (1994).

13. H. De Raedt, K. Michielsen, Comp. in Phys. 8, 600 (1994).

14. A. Rouhi, J. Wright, Comp. in Phys. 9, 554 (1995).

15. B.A. Shadwick and W.F. Buell, Phys. Rev. Lett. 79, 5189 (1997).

16. M. Krech, A. Bunker, and D.P. Landau, Comp. Phys. Comm. 111, 1 (1998).

17. P. Tran, Phys. Rev. E 58, 8049 (1998).

18. K. Michielsen, H. De Raedt, J. Przeslawski, and N. Garcia, Phys. Rep. 304, 89 (1998).

19. H. De Raedt, A.H. Hams, K. Michielsen, and K. De Raedt, Comp. Phys. Comm. 132, 1 (2000).

20. E. Yablonovitch, Phys. Rev. Lett. 58, 2059 (1987).

21. K.M. Ho, C.T. Chan and C.M. Soukoulis, Phys. Rev. Lett. 65, 3152 (1990).

22. C.M. Anderson and K.P. Giapis, Phys. Rev. Lett. 77, 2949 (1996).

23. R. Alben, M. Blume, H. Krakauer, and L. Schwartz, Phys. Rev. B 12, 4090 (1975).

24. V.G. Veselago, Sov. Phys. USPEKHI 10, 509 (1968).

25. R.A. Shelby, D.R. Smith, and S. Schultz, Science 29277 (2001). 